\title{
Pacific
}

Journal of

Mathematics

\section{SUBSEMIGROUPS OF COMPLETELY SIMPLE SEMIGROUPS}

AnNe ANTONiPPILlai AND Francis PAStiJn 


\title{
SUBSEMIGROUPS OF COMPLETELY SIMPLE SEMIGROUPS
}

\section{A. Antonippillai and Francis Pastijn}

\begin{abstract}
A semigroup which is embeddable into a completely simple semigroup satisfies some weak cancellation laws (C). Conversely, if $S$ is a semigroup which generates a variety which does not contain the variety of left regular bands nor the variety of right regular bands, then $S$ is embeddable into a completely simple semigroup if it satisfies the laws (C). This result is used to characterize the subsemigroups of completely simple semigroups over nilpotent groups of class $n$.
\end{abstract}

1. Introduction. In 1937 Malcev disproved a claim made by Sushkevich in 1935 that every cancellative semigroup can be embedded in a group [7], [17]. In fact, if can be shown that there exists a cancellative semigroup which can be embedded in a completely simple semigroup but not in a group [1, Example 1.5]. Malcev further showed in [8] that a semigroup $S$ is embeddable in a group if and only if $S$ satisfies some infinite set of implications, and in [9] he proved that there exists no finite set $\Sigma$ of implications such that $S$ is embeddable in a group if and only if $S$ satisfies the implications of $\Sigma$. It is therefore all the more surprising that the following result holds: if $S$ is a semigroup such that $S$ generates a semigroup variety which is not the variety of all semigroups, then $S$ is embeddable in a group if and only if $S$ is cancellative [10], [12].

In [1] it was observed that the class of all semigroups which are embeddable in a completely simple semigroup is definable by implications. In view of the above-mentioned results of [10] and [12] one may try to resolve the following problem: find a finite set of implications $\Sigma$ such that, given any semigroup $S$ which satisfies some nontrivial identity, then $S$ is embeddable in a completely simple semigroup if and only if $S$ satisfies the implications of $\Sigma$. In $\S 2$ we give a solution to this problem in a special case. We establish a set consisting of four weak cancellation laws (C) such that, given any semigroup $S$ which generates a variety not containing the variety of all left regular bands nor the variety of all right regular bands, then $S$ is embeddable in a completely simple semigroup if and only if $S$ satisfies the four impliciations (C). The strength of this theorem is demonstrated 
in $\S 3$ where we characterize the semigroups which are embeddable in a completely simple semigroup over nilpotent groups of class $n$ : we find semigroup identities $\left(N_{n}\right)$ and $\left(N_{n}^{\prime}\right)$ such that a semigroup $S$ is embeddable in a completely simple semigroup over nilpotent groups of class $n$ if and only if $S$ satisfies the four weak cancellation laws (C) and the identities $\left(N_{n}\right),\left(N_{n}^{\prime}\right)$.

This paper follows the notation and terminology of [2] and [14]. These references also provide for the necessary background on completely simple semigroups. We refer to [6], [15] for a treatment of completely simple semigroups from the point of view of universal algebra.

2. Weak cancellation laws. In the following we shall make use of the following implications:

$$
\begin{gathered}
x z_{1}=y z_{2}, \quad z_{3} x=z_{3} y \Rightarrow x=y, \\
z_{1} x=z_{2} y, \quad x z_{3}=y z_{3} \Rightarrow x=y, \\
z_{1} x y=z_{2} x y \Rightarrow z_{1} x=z_{2} x, \\
y x z_{1}=y x z_{2} \Rightarrow x z_{1}=x z_{2} .
\end{gathered}
$$

Since each of these implications follows from the left and right cancellation laws

$$
z x=z y \Rightarrow x=y, \quad x z=y z \Rightarrow x=y,
$$

we call the laws (C1), (C2), (C3), (C4) weak cancellation laws. We observe that $(\mathrm{C} 1)$ is the dual of $(\mathrm{C} 2)$ whereas $(\mathrm{C} 3)$ is the dual of $(\mathrm{C} 4)$. We shall denote these four weak cancellation laws by $(C)$.

We shall show that every subsemigroup of a completely simple semigroup satisfies the weak cancellation laws (C). One cannot expect that, conversely, every semigroup satisfying the laws of $(C)$ is embeddable in a completely simple semigroup. However, the main theorem of this section brings a converse in some special circumstances.

A semigroup $S$ is said to be cancellative if it satisfies the two cancellation laws (1) and $S$ is said to be reversible if for every $a, b \in S$ we have $a S \cap b S \neq \varnothing$ and $S a \cap S b \neq \varnothing$. If the semigroup $S$ is the disjoint union of its subsemigroups $S_{i \lambda}, i \in I, \lambda \in \Lambda$, such that $S_{i \lambda} S_{j \mu} \subseteq S_{i \mu}$ for all $i, j \in I, \lambda, \mu \in \Lambda$, then we say that $S$ is a rectangular band $I \times \Lambda$ of the semigroups $S_{i \lambda},(i, \lambda) \in I \times \Lambda$.

LEMMA 1. A semigroup which is a rectangular band of cancellative reversible semigroups satisfies the implications (C). 
Proof. Let $S$ be a rectangular band $I \times \Lambda$ of the cancellative reversible semigroups $S_{i \lambda},(i, \lambda) \in I \times \Lambda$, and let $a, b, c, d \in S$ such that $c a b=d a b$. Then there exists $(i, \lambda) \in I \times \Lambda$ such that $c a, d a \in S_{i \lambda}$. Let $b \in S_{j \mu}$. From $c a b=d a b$ we have that bacaba $=$ badaba. Since baca, bada, $b a \in S_{j \lambda}$, we have that baca $=b a d a$, since $S_{j \lambda}$ is cancellative. Therefore cbaca $=$ cbada . Since $c b a, c a, d a \in S_{i \lambda}$, and since $S_{i \lambda}$ is cancellative, we find $c a=d a$. Thus, from $c a b=d a b$ it follows that $c a=d a$. We have proved that $S$ satisfies (C3). By duality, $S$ satisfies (C4).

Let $a, b, c, d, e \in S$ such that $c a=d b$ and $a e=b e$. Then $d b e=c a e=c b e$ and since by the above $S$ satisfies (C3), we conclude that $c a=d b=c b$. From $c a=d b$ and $a e=b e$ it also follows that there exists $(i, \lambda) \in I \times \Lambda$ such that $a, b \in S_{i \lambda}$. Since $S_{i \lambda}$ is reversible, there exist $u, v \in S_{i \lambda}$ such that $a u=b v$. Hence $c a u=$ $c b v=c a v$. Since $S$ satisfies $(\mathrm{C} 4), c a u=c a v$ yields $a u=a v$. Since $a, b, u, v \in S_{i \lambda}$, and since $S_{i \lambda}$ is cancellative, it follows from $a u=a v$ and $a u=b v$ that $a=b$. We have proved that $S$ satisfies (C2). By duality, $S$ satisfies (C1).

Corollary 2. If the semigroup $S$ can be embedded into a completely simple semigroup, then $S$ satisfies the weak cancellation laws (C).

Proof. A completely simple semigroup is a rectangular band of groups. Therefore it satisfies the weak cancellation laws (C) by Lemma 1. Consequently every subsemigroup of a completely simple semigroup satisfies the laws $(\mathrm{C})$.

Let $S$ be a subsemigroup of a completely simple semigroup $M$. We say that $M$ is a semigroup of quotients of $S$ if $M=\left\{a b^{-1} \mid a, b \in\right.$ $S\}=\left\{b^{-1} a \mid a, b \in S\right\}$; here $b^{-1}$ denotes the inverse of $b$ within the maximal subgroup of $M$ which contains $b$. Using the results of [4], we give a proof for the following result which also appears as Theorem 4.4 in [5].

THEOREM 3. A semigroup $S$ can be embedded in a completely simple semigroup of quotients of $S$ of the form

$$
\begin{aligned}
M & =\left\{a b^{-1} \mid a, b \in S, a \mathscr{L} b \text { in } M\right\} \\
& =\left\{b^{-1} a \mid a, b \in S, a \mathscr{R} b \text { in } M\right\}
\end{aligned}
$$

if and only if $S$ is a rectangular band of cancellative reversible semigroups. 
Proof. Let $S$ be a rectangular band $I \times \Lambda$ of cancellative reversible semigroups $S_{i \lambda},(i, \lambda) \in I \times \Lambda$. We shall show that the relation $\rho$ on $S$ which is defined by $a \rho b$ if and only if $a S \cap b S \neq \varnothing$, is a congruence relation. This will follow if we show that for all $a, b, u, v \in S$,

$$
a S \cap b S \neq \varnothing \Rightarrow a u S \cap b v S \neq \varnothing .
$$

Indeed, if $a S \cap b S \neq \varnothing$, then $a \in S_{i \lambda}, b \in S_{i \mu}$ for some $i \in I$ and $\lambda, \mu \in \Lambda$. Let $u, v$ be any elements of $S$. Then $a u a, b v a \in S_{i \lambda}$ and since $S_{i \lambda}$ is reversible, there exist $s, t \in S_{i \lambda}$ such that auas = bvat. It follows that (3) holds. By duality, for all $a, b, u, v \in S$

$$
S a \cap S b \neq \varnothing \Rightarrow S u a \cap S v b \neq \varnothing .
$$

By Lemma 1, $S$ satisfies the implications (C1) and (C2). By Theorem 6.1 of [4], $S$ can be embedded into a completely simple semigroup $M$ of quotients of $S$ which by Lemmas 2.10, 4.18 and 4.20 of [4] is of the form (2).

Conversely, let $M$ be a completely simple semigroup of quotients of $S$ of the form (2). Then $M$ is a rectangular band $I \times \Lambda$ of groups $G_{i \lambda},(i, \lambda) \in I \times \Lambda$, and for each $(i, \lambda) \in I \times \Lambda, S_{i \lambda}=G_{i \lambda} \cap S$ is a cancellative semigroup. Let $a, b \in S_{i \lambda}$. Then $a^{2} b^{-1}=c^{-1} d$ for some $d \in S_{i \lambda}$ and $c \in S_{i \mu}, \mu \in \Lambda$. Then $c a^{2}=c c^{-1} d b=d b$, where $a, b, c a, d \in S_{i \lambda}$. Hence $S_{i \lambda} a \cap S_{i \lambda} b \neq \varnothing$. In a dual way one shows that $a S_{i \lambda} \cap b S_{i \lambda} \neq \varnothing$. Therefore the $S_{i \lambda},(i, \lambda) \in I \times \Lambda$, are reversible.

The above theorem is the analogue for completely simple semigroups of corresponding theorems by Ore and Dubreil for groups [3], [13] (see also Theorems 1.23 and 1.24 of [2]).

We now set out to prove a converse of Corollary 2 for semigroups which satisfy a certain type of identity. Let $X$ be any alphabet and $u$ a nonempty word in the free semigroup $X^{+}$on $X$. Let $x_{1}, \ldots, x_{n}$ be the letters which occur in $u$ and suppose that $u=x_{1} u_{1} x_{2} u_{2} \cdots x_{n} u_{n}$ for some $u_{1}, \ldots, u_{n}$ in the free monoid $X^{*}$ such that for every $1<$ $i \leq n, x_{i}$ does not occur in $x_{1} u_{1} \cdots x_{i-1} u_{i-1}$. Then we use the notation $i(u)=x_{1} x_{2} \cdots x_{n}$. If $\bar{u}$ denotes the mirror image of $u$, then we put $f(u)=i(\bar{u})$. It follows from the results of Section II.3 of [14] that $\Sigma_{i}=\left\{u=v \mid u, v \in X^{+}, i(u)=i(v)\right\}$ is the set of all identities satisfied by all left regular bands, and $\Sigma_{f}=\{u=v \mid u, v \in$ $\left.X^{+}, f(u)=f(v)\right\}$ the set of all identities satisfied by all right regular bands.

THEOREM 4. Let $S$ be a semigroup which generates a variety which does not contain the variety of all left regular bands nor the variety of 
all right regular bands. Then $S$ is a rectangular band of cancellative reversible semigroups if and only if $S$ satisfies the weak cancellation laws $(\mathrm{C})$.

Proof. If $S$ is a rectangular band of cancellative reversible semigroups, then $S$ satisfies the laws (C) by Lemma 1.

Conversely, let $S$ satisfy the weak cancellation laws (C). Since the variety generated by $S$ does not contain the variety of all left regular bands, $S$ satisfies an identity $u=v$ with $i(u) \neq i(v)$. It is then easy to see that $S$ satisfies an identity of the form $x^{n} z u_{1}=x^{m} y v_{1}$, with $m, n \geq 1$ and $u_{1}$ and $v_{1}$ words in which only the letters $x$, $y$ and $z$ occur. Substituting $x$ by $x, z$ by $x^{m} z$ and $y$ by $x^{n} y$ in this identity, we obtain an identity of the form $x^{n+m} z u_{2}=x^{n+m} y v_{2}$ which is satisfied in $S$. Since $S$ satisfies (C3) we find that $S$ satisfies an identity of the form $x z u_{2}=x y v_{2}$. In a dual way we can prove that $S$ satisfies an identity of the form $u_{3} z x=v_{3} y x$ where in $u_{3}$ and $v_{3}$ only the letters $x, y$ and $z$ occur.

On $S$ we define a relation $\beta$ by the following. For $a, b \in S$ we put

$$
a \beta b \Leftrightarrow a p=b q, p^{\prime} a=q^{\prime} b \quad \text { for some } p, q, p^{\prime}, q^{\prime} \in S .
$$

It is obvious that $\beta$ is a reflexive and symmetric relation. Let $a \beta b \beta c$. Then for some $p, q, s, t \in S$ we have that $a p=b q$ and $b s=c t$. Substituting $x$ by $b, z$ by $q$ and $y$ by $s$ in the identity $x z u_{2}=x y v_{2}$ we find that $b q m=b s n$ in $S$ for some $m, n \in S$. Therefore $a p m=b q m=b s n=c t n$. From this and the result of a dual argument we conclude that $a \beta c$. Thus $\beta$ is an equivalence relation.

Let $a \beta b$ and let $p, q, p^{\prime}$ and $q^{\prime}$ be as in (5). Then certainly $p^{\prime} a s=q^{\prime} b s$. Substituting $x$ by $a, z$ by $s$ and $y$ by $p$ in $x z u_{2}=$ $x y v_{2}$ we find that asm =apn=bqn in $S$ for some $m, n \in S$. Substituting $x$ by $b, z$ by $q n$ and $y$ by $s$ in $x z u_{2}=x y v_{2}$ we obtain $b q n c=b s d$ in $S$ for some $c, d \in S$. Thus $a s m c=b q n c=b s d$ and we can conclude that as $\beta b s$. A dual argument gives $s a \beta s b$. Therefore $\beta$ is a congruence. Since for every $a, b \in S$ we obviously have $a \beta a^{2}$ and $a \beta a b a$, it follows that $\beta$ is a rectangular band congruence.

Let $a \beta b$ and suppose that $a c=b c$. There exist $p^{\prime}, q^{\prime} \in S$ such that $p^{\prime} a=q^{\prime} b$. By (C2) we thus have $a=b$. This and a dual argument show that each $\beta$-class is cancellative. Each $\beta$-class is a semigroup which does not generate the variety of all semigroups. Hence by 
a result of [10] or [12], each $\beta$-class $a \beta$ can be embedded in a group of quotients $\left\{c d^{-1} \mid c, d \in a \beta\right\}=\left\{c^{-1} d \mid c, d \in a \beta\right\}$. Using Dubreil's theorem [3] (Theorem 1.24 in [2]), we have that each $\beta$-class is reversible.

THEOREM 5. Let $S$ be a semigroup which generates a variety which does not contain the variety of all left regular bands nor the variety of all right regular bands. Then $S$ can be embedded in a completely simple semigroup if and only if $S$ satisfies the weak cancellation laws (C). If this is the case, then $S$ can be embedded in a completely simple. semigroup of quotients of $S$ of the form (2).

Proof. The proof follows immediately from Corollary 2, Theorem 3 and Theorem 4.

REMARK. Theorem 5 is nontrivial only if the variety generated by $S$ contains the variety of all commutative semigroups. If not, then $S$ is periodic, that is, $S$ satisfies an identity of the form $x^{m}=x^{m+n}$, $m, n \geq 1$; it is well known that a periodic semigroup $S$ can be embedded in a completely simple semigroup if and only if $S$ is completely simple.

If $S$ satisfies the weak cancellation laws (C) and an identity of the form $u=v$, where the first letter in $u$ differs from the first letter in $v$ and the last letter in $u$ differs from the last letter in $v$, then the conditions of Theorem 5 are satisfied and so $S$ can be embedded in a completely simple semigroup $M$. One easily sees that the completely simple subsemigroup of $M$ generated by $S$ is in fact a group. Thus, if $S$ satisfies the weak cancellation laws (C) and an identity $u=v$ of the above form, then $S$ is embeddable in a group. In particular, if $S$ is a commutative semigroup satisfying the weak cancellation laws (C), then $S$ can be embedded in an abelian group.

3. Subsemigroups of completely simple semigroups over nilpotent groups. A nilpotent group is said to be of class $n$ if the length of its ascending central series is at most $n$. The class of all nilpotent groups of class $n, n \geq 1$, constitutes a subvariety $\mathbf{N}_{n}$ of the variety $\mathbf{G}$ of all group varieties (see e.g. [11]). In [12] it is shown that a group is in $\mathbf{N}_{n}$ if and only if it satisfies some semigroup identity $\left(L_{n}\right)$. These semigroup identities are given by the following: first define the $q_{i}$, $1 \leq i \leq n$, inductively by

$$
q_{1}\left(x, y, z_{1}, \ldots, z_{n-1}\right)=x y,
$$


(7)

$$
\begin{aligned}
& q_{i+1}\left(x, y, z_{1}, \ldots, z_{n-1}\right) \\
& \quad=q_{i}\left(x, y, z_{1}, \ldots, z_{n-1}\right) z_{i} q_{i}\left(y, x, z_{1}, \ldots, z_{n-1}\right), \\
& 1 \leq i \leq n-1,
\end{aligned}
$$

and then $\left(L_{n}\right)$ is given by

$$
\left(L_{n}\right) \quad q_{n}\left(x, y, z_{1}, \ldots, z_{n-1}\right)=q_{n}\left(y, x, z_{1}, \ldots, z_{n-1}\right) .
$$

One sees that the only variables occurring in $q_{i+1}, i \geq 1$, are $x, y$ and $z_{1}, \ldots, z_{i}$.

For a completely simple semigroup $S$ and $a \in S$ we may denote the inverse of $a$ within the maximal subgroup of $S$ containing $a$ by $a^{-1}$. As such, completely simple semigroups are semigroups endowed with a unary operation. Viewed in this way, the class of all completely simple semigroups forms a variety (see e.g. [15]). For any group variety $\mathbf{V}$, let $\mathbf{C S}(\mathbf{V})$ be the class of all completely simple semigroups whose maximal subgroups are in $\mathbf{V}$. It is easy to see that $\mathbf{C S}(\mathbf{V})$ is a subvariety of the variety $\mathbf{C S}$ of all completely simple semigroups [15]: indeed, if $\mathbf{V}$ is given by the set $\Sigma=\left\{u_{i}\left(x_{1}, \ldots, x_{n_{i}}\right)=v_{i}\left(x_{1}, \ldots, x_{n_{i}}\right) \mid i \in I\right\}$ of identities, then $\mathbf{C S}(\mathbf{V})$ is determined by the set of identities $\Sigma^{\prime}=$ $\left\{u_{i}\left(t x_{1} t, \ldots, t x_{n_{i}} t\right)=v_{i}\left(t x_{1} t, \ldots, t x_{n_{t}} t\right) \mid i \in I\right\}$. In particular, if $\Sigma$ consists of semigroup identities only, then $\Sigma^{\prime}$ consists of semigroup identities only. We shall say that $\mathbf{C S}(\mathbf{V})$ consists of completely simple semigroups over V-groups.

By the preceding, the variety $\mathbf{C S}\left(\mathbf{N}_{n}\right)$ of completely simple semigroups over nilpotent groups of class $n$ is determined by the semigroup identity

$$
\begin{aligned}
& \left(L_{n}^{\prime}\right) \quad q_{n}\left(t x t, t y t, t z_{1} t, \ldots, t z_{n-1} t\right) \\
& \quad=q_{n}\left(t y t, t x t, t z_{1} t, \ldots, t z_{n-1} t\right),
\end{aligned}
$$

so that every semigroup which is embeddable into a completely simple semigroup over nilpotent groups of class $n$ satisfies $\left(L_{n}^{\prime}\right)$ and the weak cancellation laws $(\mathrm{C})$. Conversely, a semigroup which satisfies $\left(L_{n}^{\prime}\right)$ and the weak cancellation laws $(\mathrm{C})$ can be embedded in a completely simple semigroup by Theorem 5 . Unfortunately we have no guarantee that this completely simple semigroup satisfies $\left(L_{n}^{\prime}\right)$ and consequently we do not know whether the maximal subgroups of this completely simple semigroup are nilpotent of class $n$. Therefore, if we want to characterize the subsemigroups of members of $\mathbf{C S}\left(\mathbf{N}_{n}\right)$, we need a new approach. 
We define the semigroup words $p_{i}$ and $p_{i}^{\prime}, 1 \leq i \leq n$, inductively by

$$
p_{1}=x x y x, \quad p_{1}^{\prime}=x y x x
$$

$$
\begin{aligned}
& p_{i+1}\left(x, y, z_{1}, \ldots, z_{n-1}\right) \\
& \quad=p_{i}\left(x, y, z_{1}, \ldots, z_{n-1}\right) z_{i} p_{i}^{\prime}\left(x, y, z_{1}, \ldots, z_{n-1}\right), \\
& \begin{array}{l}
p_{i+1}^{\prime}\left(x, y, z_{1}, \ldots, z_{n-1}\right) \\
\quad=p_{i}^{\prime}\left(x, y, z_{1}, \ldots, z_{n-1}\right) z_{i} p_{i}\left(x, y, z_{1}, \ldots, z_{n-1}\right), \\
\quad 1 \leq i \leq n-1,
\end{array}
\end{aligned}
$$

and then $\left(N_{n}\right)$ is given by

$$
\left(N_{n}\right) \quad p_{n}\left(x, y, z_{1}, \ldots, z_{n-1}\right)=p_{n}^{\prime}\left(x, y, z_{1}, \ldots, z_{n-1}\right) .
$$

Again, for $i \geq 1$, the only variables occurring in $p_{i+1}$ and $p_{i+1}^{\prime}$ are $x, y$ and $z_{1}, \ldots, z_{i}$.

We first show that for groups the identity $\left(N_{n}\right)$ serves the same purpose as the identity $\left(L_{n}\right)$. The following mimics the corresponding lemma and its proof of [12].

LeMmA 6. Let $G$ be a group and $S$ a subsemigroup of $G$ such that $S$ satisfies the identity $\left(N_{n}\right)$ and $G=\left\{a b^{-1} \mid a, b \in S\right\}$. Then $G$ is nilpotent of class $n$.

Proof. We give a proof by induction on $n$. If $S$ satisfies $N_{1}$, then $S$ is commutative since $S$ is cancellative. Therefore $G$ is an abelian group, that is, a nilpotent group of class 1 .

We now assume that $n>1$, and that $\left(N_{n}\right)$ is satisfied in $S$. Thus,

$$
p_{n-1} z_{n-1} p_{n-1}^{\prime}=p_{n-1}^{\prime} z_{n-1} p_{n-1}
$$

is satisfied in $S$ and so

$$
p_{n-1}^{\prime-1} p_{n-1} z_{n-1} p_{n-1}^{\prime}=z_{n-1} p_{n-1}^{\prime} p_{n-1}^{\prime-1} p_{n-1}
$$

is satisfied in $G$ whenever the $x, y, z_{1}, \ldots, z_{n-1}$ are substituted by elements of $S$. Let us substitute $x, y, z_{1}, \ldots, z_{n-2}$ by $c, d, e_{1}$, $\ldots, e_{n-2} \in S$, respectively and use the notation

$$
p_{n-1}\left(c, d, e_{1}, \ldots, e_{n-2}\right)=s, \quad p_{n-1}^{\prime}\left(c, d, e_{1}, \ldots, e_{n-2}\right)=s^{\prime}
$$

By (11) we see that for all $a, b \in S$, as and $b s^{\prime}$ commute with $s^{\prime-1} s$, whence $a b^{-1}=a s^{\prime}\left(b s^{\prime}\right)^{-1}$ commutes with $s^{-1} s$. Since $G=$ $\left\{a b^{-1} \mid a, b \in S\right\}$, it follows that $s^{-1} s$ is in the center $C$ of $G$, whence 
$s C=s^{\prime} C$ in $G / C$. This entails that the subsemigroup $T=\{a C \mid a \in$ $S\}$ of $G / C$ satisfies $\left(N_{n-1}\right)$. Since $G / C=\left\{(a C)(b C)^{-1} \mid a C, b C \in\right.$ $T\}$ we may apply the induction hypothesis and we conclude that $G / C$ is nilpotent of class $n-1$. Hence $G$ is nilpotent of class $n$.

THEOREM 7. The semigroup $S$ can be embedded in a nilpotent group of class $n$ if and only if it is cancellative and satisfies the law $\left(N_{n}\right)$.

Proof. Let $S$ be a subsemigroup of a nilpotent group $G$ of class $n$. We shall show that $G$ satisfies $\left(N_{n}\right)$. We show this by induction on $n$. If $n=1$, then $G$ is abelian and so $G$ satisfies $\left(N_{1}\right)$. Let $n>1$ and let $C$ be the center of $G$. By the induction hypothesis we know that $G / C$ satisfies $\left(N_{n-1}\right)$. Let us substitute the variables $x, y, z_{1}, \ldots, z_{n-2}$ by $c, d, e_{1}, \ldots, e_{n-2} \in G$, respectively, and we use the notation (12). Then $s C=s^{\prime} C$ so that $s s^{\prime-1}$ belongs to $C$; in particular, $s s^{\prime-1}$ commutes with $s^{-1}$, whence $s s^{-1} s^{-1} s^{\prime}=s^{-1} s s^{\prime-1} s^{\prime}=1_{G}$, from which we have that $s s^{\prime-1}=\left(s^{-1} s^{\prime}\right)^{-1}=s^{\prime-1} s$. If $a \in G$ we thus have that $s^{\prime-1} s a=a s s^{\prime-1}$ or $s a s^{\prime}=s^{\prime} a s$. Thus $\left(N_{n}\right)$ is satisfied in $G$. It now follows that every subsemigroup of the group $G$ is cancellative and satisfies $\left(N_{n}\right)$.

Conversely, let $S$ be a cancellative semigroup which satisfies $\left(N_{n}\right)$. By [10] or [12] $S$ can be embedded into a group $G=\left\{a b^{-1} \mid a, b \in S\right\}$ and by Lemma $6 G$ is nilpotent of class $n$.

COROLlaRY 8. $\mathbf{N}_{n}$ is the group variety determined by $\left(N_{n}\right)$.

THEOREM 9. $\mathbf{C S}\left(\mathbf{N}_{n}\right)$ is the variety of completely simple semigroups determined by $\left(N_{n}\right)$.

Proof. Let $M$ be a completely simple semigroup which satisfies $\left(N_{n}\right)$. Since the maximal subgroups of $M$ satisfy $\left(N_{n}\right)$, they must be nilpotent of class $n$ by Corollary 8 . Hence $M \in \mathbf{C S}\left(\mathbf{N}_{n}\right)$.

Conversely, let $M \in \mathbf{C S}\left(\mathbf{N}_{n}\right)$. We shall substitute the variables $x, y, z_{1}, \ldots, z_{n-1}$ which occur in $\left(N_{n}\right)$ by $c, d, e_{1}, \ldots, e_{n-1} \in M$, respectively. We can construct a Rees matrix representation for $M$ and we shall henceforth identify $M$ with this Rees matrix semigroup $\mathscr{M}(G ; I, \Lambda ; P)$, where $P=\left(p_{\lambda i}\right)$. Accordingly, $c=\left(i_{c}, g_{c}, \lambda_{c}\right), d=$ $\left(i_{d}, g_{d}, \lambda_{d}\right), e_{j}=\left(i_{j}, g_{j}, \lambda_{j}\right), 1 \leq j \leq n-1$. We can always assume that $p_{\lambda_{c} i}=p_{\lambda_{c}}=1$ for every $i \in I$ and every $\lambda \in \Lambda$. Observe that in both $p_{n}$ and $p_{n}^{\prime}$ each occurrence of $y, z_{1}, \ldots, z_{n-1}$ is preceded and followed by an occurrence of $x$. Also $x$ is the first and last letter in 
both $p_{n}$ and $p_{n}^{\prime}$. Therefore, and in view of the way in which $P$ has been normalized, we have that

$$
p_{n}\left(c, d, e_{1}, \ldots, e_{n-1}\right)=\left(i_{c}, p_{n}\left(g_{c}, g_{d}, g_{1}, \ldots, g_{n-1}\right), \lambda_{c}\right)
$$

and

$$
p_{n}^{\prime}\left(c, d, e_{1}, \ldots, e_{n-1}\right)=\left(i_{c}, p_{n}^{\prime}\left(g_{c}, g_{d}, g_{1}, \ldots, g_{n-1}\right), \lambda_{c}\right) .
$$

Since $G$ satisfies $p_{n}=p_{n}^{\prime}$ if follows that

$$
p_{n}\left(c, d, e_{1}, \ldots, e_{n-1}\right)=p_{n}^{\prime}\left(c, d, e_{1}, \ldots, e_{n-1}\right)
$$

and consequently $M$ satisfies $\left(N_{n}\right)$.

We shall now define the semigroup words $r_{i}$ and $r_{i}^{\prime}, 1 \leq i \leq n$, inductively by

$$
r_{1}=x t x y x, \quad r_{1}^{\prime}=x y x t x
$$

$$
\begin{aligned}
& r_{i+1}\left(x, y, t, z_{1}, \ldots, z_{n-1}\right) \\
& \quad=r_{i}\left(x, y, t, z_{1}, \ldots, z_{n-1}\right) z_{i} r_{i}^{\prime}\left(x, y, t, z_{1}, \ldots, z_{n-1}\right), \\
& r_{i+1}^{\prime}\left(x, y, t, z_{1}, \ldots, z_{n-1}\right) \\
& \quad=r_{i}^{\prime}\left(x, y, t, z_{1}, \ldots, z_{n-1}\right) z_{i} r_{i}\left(x, y, t, z_{1}, \ldots, z_{n-1}\right), \\
& 1 \leq i \leq n-1,
\end{aligned}
$$

and then $\left(N_{n}^{\prime}\right)$ is given by

$$
\left(N_{n}^{\prime}\right) \quad r_{n}\left(x, y, t, z_{1}, \ldots, z_{n-1}\right)=r_{n}^{\prime}\left(x, y, t, z_{1}, \ldots, z_{n-1}\right) .
$$

For any $i \geq 1$, the only variables occurring in $r_{i+1}$ and $r_{i+1}^{\prime}$ are $x$, $y, t$ and $z_{1}, \ldots, z_{i}$.

LEMMA 10. A semigroup which can be embedded in a member of $\mathbf{C S}\left(\mathbf{N}_{n}\right)$ satisfies $\left(N_{n}^{\prime}\right)$.

Proof. Let $G$ be a nilpotent group of class $n$. We shall show by induction on $n$ that $G$ satisfies $\left(N_{n}^{\prime}\right)$. If $n=1$, then $G$ is abelian and so $G$ satisfies $\left(N_{1}^{\prime}\right)$ in this case. If $n>1$, we let $C$ be the center of $G$ and we may assume by the induction hypothesis that $G / C$ satisfies $\left(N_{n-1}^{\prime}\right)$. We substitute the variables $x, y, t, z_{1}, \ldots, z_{n-2}$ by $c, d, f, e_{1}, \ldots, e_{n-2} \in G$, respectively and we use the notation? $r_{n-1}\left(c, d, f, e_{1}, \ldots, e_{n-2}\right)=s, \quad r_{n-1}^{\prime}\left(c, d, f, e_{1}, \ldots, e_{n-2}\right)=s^{\prime}$. As in the proof of Theorem 7 we can show that for every $a \in G$, $s a s^{\prime}=s^{\prime}$ as, thus $\left(N_{n}^{\prime}\right)$ is satisfied in $G$. 
We see that in both $r_{n}$ and $r_{n}^{\prime}$ each occurrence of $y, t, z_{1}, \ldots$, $z_{n-1}$ is preceded and followed by an occurrence of $x$. Using an argument as in the proof of the converse part of Theorem 9 we can then show that $\left(N_{n}^{\prime}\right)$ is satisfied in every member of $\mathbf{C S}\left(\mathbf{N}_{n}\right)$. Consequently $\left(N_{n}^{\prime}\right)$ is satisfied in every subsemigroup of a member of $\mathbf{C S}\left(\mathbf{N}_{n}\right)$.

THEOREM 11. A semigroup $S$ can be embedded in a completely simple semigroup whose maximal subgroups are nilpotent of class $n$ if and only if $S$ satisfies $\left(N_{n}\right),\left(N_{n}^{\prime}\right)$, and the weak cancellation laws $(\mathrm{C})$.

Proof. If $S$ can be embedded in a member of $\mathbf{C S}\left(\mathbf{N}_{n}\right)$, then $S$ satisfies the weak cancellation laws (C) by Corollary 2, and $S$ satisfies $\left(N_{n}\right)$ and $\left(N_{n}^{\prime}\right)$ by Theorem 9 and Lemma 10.

Conversely, let $S$ satisfy $\left(N_{n}\right),\left(N_{n}^{\prime}\right)$ and the weak cancellation laws $(\mathrm{C})$. We see that $i\left(r_{n}\right)=x t y z_{1} \cdots z_{n-1}, i\left(r_{n}^{\prime}\right)=x y t z_{1} \cdots z_{n-1}$, $f\left(r_{n}\right)=x y t z_{1} \cdots z_{n-1}$ if $n$ is odd, $f\left(r_{n}\right)=x t y z_{1} \cdots z_{n-1}$ if $n$ is even, $f\left(r_{n}^{\prime}\right)=x t y z_{1} \cdots z_{n-1}$ if $n$ is odd, $f\left(r_{n}^{\prime}\right)=x y t z_{1} \cdots z_{n-1}$ if $n$ is even. Then by Theorem $5, S$ can be embedded into a completely simple semigroup $M=\left\{a b^{-1} \mid a, b \in S, a \mathscr{L} b\right.$ in $\left.M\right\}$. We can construct a Rees matrix representation for $M$ and we shall identify $M$ with this Rees matrix semigroup $\mathscr{M}(G ; I, \Lambda ; P)$. We can moreover assume that $1 \in I \cap \Lambda$ and that $p_{\lambda 1}=p_{1 i}=1_{G}$ for all $i \in I$ and $\lambda \in \Lambda$. We put

$$
\begin{aligned}
G_{11} & =\{(1, g, 1) \mid g \in G\}, \\
S_{1} & =\{(i, g, 1) \mid g \in G, i \in I\} \cap S, \\
T_{11} & =\left\{(1, t, 1) \in G_{11} \mid(i, t, 1) \in S_{1} \text { for some } i \in I\right\} .
\end{aligned}
$$

Then $S_{1}$ is a subsemigroup of $S$ and $S_{1} \rightarrow T_{11},(i, t, 1) \rightarrow(1, t, 1)$ is a surjective homomorphism. Therefore $T_{11}$ satisfies $\left(N_{n}\right)$. By the above, every element in $G_{11}$ can be written in the form

$$
(1, s, 1)(i, t, 1)^{-1}=\left(1, s t^{-1}, 1\right)
$$

for some $(1, s, 1),(i, t, 1) \in S_{1}$. Hence every element of $G_{11}$ is of the form $(1, s, 1)(1, t, 1)^{-1}$ for some $(1, s, 1),(1, t, 1) \in T_{11}$. By Lemma 6 we then have that $G \cong G_{11}$ is a nilpotent group of class $n$. Consequently the maximal subgroups of $M$ are nilpotent of class $n$.

From Theorem 5, the proofs of Theorems 7 and 11 and using duality we have: 
Corollary 12. (i) A semigroup $S$ satisfies the equivalent conditions of Theorem 7 if and only if $S$ can be embedded into a group of quotients $G=\left\{a b^{-1} \mid a, b \in S\right\}=\left\{a^{-1} b \mid a, b \in S\right\} \in \mathbf{N}_{n}$.

(ii) A semigroup $S$ satisfies the equivalent conditions of Theorem 11 if and only if $S$ can be embedded into a completely simple semigroup of quotients $M=\left\{a b^{-1} \mid a, b \in S, a \mathscr{L} b\right.$ in $\left.M\right\}=\left\{a^{-1} b \mid a, b \in S\right.$, $a \mathscr{R} b$ in $M\} \in \mathbf{C S}\left(\mathbf{N}_{n}\right)$.

Theorem 7 is equivalent to the main result of [12] which uses $\left(L_{n}\right)$ instead of $\left(N_{n}\right)$. The identity $\left(N_{n}\right)$ is more complicated than the identity $\left(L_{n}\right)$, but both turn out to be equivalent under the presence of the cancellation laws. This is not the case any more if we replace the cancellation laws by the weak cancellation laws (C).

The following special case of Theorem 11 is an analogue of a result in [16] and of Theorem 7.1 of [4] (see also [14], III.4).

THEOREM 13. A semigroup $S$ can be embedded in a completely simple semigroup whose maximal subgroups are abelian if and only if $S$ satisfies $x x y x=x y x x, x t x y x=x y x t x$ and the weak cancellation laws (C).

\section{REFERENCES}

[1] K. Byleen, and F. Pastijn, Implications for semigroups embeddable in orthocryptogroups, Rocky Mountain J. Math., 17 (1987), 463-478.

[2] A. H. Clifford, and G. B. Preston, The Algebraic Theory of Semigroups, Math. Surveys 7, Amer. Math. Soc., Providence, R.I., Vol. I, 1961, Vol. II, 1967.

[3] P. Dubreil, Sur les problèmes d'immersion et la théorie des modules, C. R. Acad. Sci. Paris, 216 (1943), 625-627.

[4] J. Fountain and M. Petrich, Completely 0-simple semigroups of quotients, J. Algebra, 101 (1986), 365-402.

[5] - Completely 0-simple semigroups of quotients III, Math. Proc. Camb. Phil. Soc., 105 (1989), 263-275.

[6] P. R. Jones, Completely simple semigroups; free products, free semigroups and varieties, Proc. Royal Soc. Edinburgh, 88A (1981), 293-313.

[7] A. Malcev, On the immersion of an algebraic ring into a field, Math. Ann., 113 (1937), 686-691.

[8] _ O vklyuchenii assotsiativnykh sistem v gruppy, Mat. Sb., 6 (48) (1939), 331-336.

[9] — O vklyuchenii assotsiativnykh sistem v gruppy, II, Mat. Sb., 8 (50) (1940), 251-264.

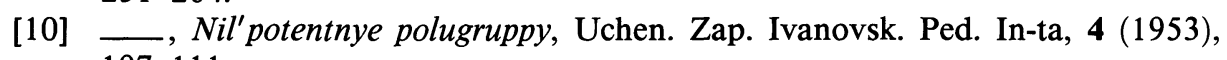
107-111.

[11] H. Neumann, Varieties of Groups, Springer-Verlag, Berlin, 1967. 
[12] B. H. Neumann and T. Taylor, Subsemigroups of nilpotent groups, Proc. Royal Soc. London, Ser. A, 274 (1963), 1-4.

[13] O. Ore, Linear equations in non-commutative fields, Ann. of Math., 32 (1931), 463-477.

[14] M. Petrich, Lectures in Semigroups, Akademie-Verlag, Berlin, 1977.

[15] M. Petrich and N. R. Reilly, Varieties of groups and of completely simple semigroups, Bull. Austral. Math. Soc., 23 (1981), 339-359.

[16] J. J. Streilein, An embedding theorem for matrices of commutative cancellative semigroups, Trans. Amer. Math. Soc., 208 (1975), 127-140.

[17] A. Sushkevich, Pro poshirennya pivgrupy do tsiloi grupy, Zapiski Khar'kovskogo Matematicheskogo Tovarishchestva, Ser. 4, 12 (1935), 81-87.

Received May 30, 1991 and in revised form September 9, 1991.

MARQUETTE UNIVERSITY

MilWAUKeE, WI 53233 



\title{
PACIFIC JOURNAL OF MATHEMATICS \\ Founded by \\ E. F. BeCKenbach (1906-1982) F. Wolf (1904-1989)
}

\section{EDITORS}

\author{
V. S. VARADARAJAN \\ (Managing Editor) \\ University of California \\ Los Angeles, CA 90024-1555 \\ vsv@math.ucla.edu \\ Herbert Clemens \\ University of Utah \\ Salt Lake City, UT 84112 \\ clemens@math.utah.edu \\ F. Michael Christ \\ University of California \\ Los Angeles, CA 90024-1555 \\ christ@math.ucla.edu \\ THOMAS ENRIGHT \\ University of California, San Diego \\ La Jolla, CA 92093 \\ tenright@ucsd.edu
}

\author{
Nicholas ERcolani \\ University of Arizona \\ Tucson, AZ 85721 \\ ercolani@math.arizona.edu \\ R. FINN \\ Stanford University \\ Stanford, CA 94305 \\ finn@gauss.stanford.edu \\ VAughan F. R. Jones \\ University of California \\ Berkeley, CA 94720 \\ vfr@math.berkeley.edu \\ STEVEN KeRCKHOFF \\ Stanford University \\ Stanford, CA 94305 \\ spk@gauss.stanford.edu
}

\author{
C. C. MOORE \\ University of California \\ Berkeley, CA 94720
}

MARTIN SCHARLEMANN

University of California

Santa Barbara, CA 93106

mgscharl@henri.ucsb.edu

\author{
HAROLD STARK \\ University of California, San Diego \\ La Jolla, CA 92093
}

\section{SUPPORTING INSTITUTIONS}

UNIVERSITY OF ARIZONA
UNIVERSITY OF BRITISH COLUMBIA
CALIFORNIA INSTITUTE OF TECHNOLOGY
UNIVERSITY OF CALIFORNIA
MONTANA STATE UNIVERSITY
UNIVERSITY OF NEVADA, RENO
NEW MEXICO STATE UNIVERSITY
OREGON STATE UNIVERSITY

\author{
UNIVERSITY OF OREGON \\ UNIVERSITY OF SOUTHERN CALIFORNIA \\ STANFORD UNIVERSITY \\ UNIVERSITY OF HAWAII \\ UNIVERSITY OF TOKYO \\ UNIVERSITY OF UTAH \\ WASHINGTON STATE UNIVERSITY \\ UNIVERSITY OF WASHINGTON
}

The Supporting Institutions listed above contribute to the cost of publication of this Journal, but they are not owners or publishers and have no responsibility for its content or policies.

\begin{abstract}
Mathematical papers intended for publication in the Pacific Journal of Mathematics should be in typed form or offset-reproduced (not dittoed), double spaced with large margins. Please do not use built up fractions in the text of the manuscript. However, you may use them in the displayed equations. Underline Greek letters in red, German in green, and script in blue. The first paragraph must be capable of being used separately as a synopsis of the entire paper. In particular it should contain no bibliographic references. Please propose a heading for the odd numbered pages of less than 35 characters. Manuscripts, in triplicate, may be sent to any one of the editors. Please classify according to the 1991 Mathematics Subject Classification scheme which can be found in the December index volumes of Mathematical Reviews. Supply name and address of author to whom proofs should be sent. All other communications should be addressed to the managing editor, or Elaine Barth, University of California, Los Angeles, California 90024-1555.

There are page-charges associated with articles appearing in the Pacific Journal of Mathematics. These charges are expected to be paid by the author's University, Government Agency or Company. If the author or authors do not have access to such Institutional support these charges are waived. Single authors will receive 50 free reprints; joint authors will receive a total of 100 free reprints. Additional copies may be obtained at cost in multiples of 50 .
\end{abstract}

The Pacific Journal of Mathematics (ISSN 0030-8730) is published monthly except for July and August. Regular subscription rate: $\$ 190.00$ a year (10 issues). Special rate: $\$ 95.00$ a year to individual members of supporting institutions.

Subscriptions, orders for numbers issued in the last three calendar years, and changes of address should be sent to Pacific Journal of Mathematics, P.O. Box 969, Carmel Valley, CA 93924, U.S.A. Old back numbers obtainable from Kraus Periodicals Co., Route 100, Millwood, NY 10546.

The Pacific Journal of Mathematics at P.O. Box 969, Carmel Valley, CA 93924 (ISSN 0030-8730) is published monthly except for July and August. Second-class postage paid at Carmel Valley, California 93924, and additional mailing offices. Postmaster: send address changes to Pacific Journal of Mathematics, P.O. Box 969, Carmel Valley, CA 93924.

\section{PUBLISHED BY PACIFIC JOURNAL OF MATHEMATICS, A NON-PROFIT CORPORATION} Copyright (C) 1992 by Pacific Journal of Mathematics 


\section{PACIFIC JOURNAL OF MATHEMATICS}

Volume $156 \quad$ No. $2 \quad$ December 1992

Surfaces in the 3-dimensional Lorentz-Minkowski space satisfying 201 $\Delta x=A x+B$

Luis Alías, Angel FerRandez and Pascual LuCAS

Lie algebras of type $D_{4}$ over number fields

209

BRUCE ALLISON

Subsemigroups of completely simple semigroups

251

Anne Antonippillai and Francis PAStiJn

Studying links via closed braids. VI. A nonfiniteness theorem

JOAN Birman and William W. MENASCO

Minimal orbits at infinity in homogeneous spaces of nonpositive curvature

MARÍA J. DRUETTA

Generalized horseshoe maps and inverse limits

SARAH ElizABETH Holte

Determinantal criteria for transversality of morphisms

DAN LAKSOV and ROBERT SPEISER

Four dodecahedral spaces

PETER LORIMER

Semifree actions on spheres

MONICA NiCOLAU

Conformal deformations preserving the Gauss map

ENALDO VERGASTA

Hecke eigenforms and representation numbers of arbitrary rank lattices 371

LYNNE WALLING 